\title{
EXCITON PROPERTIES IN CdTe/CdMnTe QUANTUM WELL STRUCTURES WITH STRONG LOCALIZATION EFFECTS
}

M. GodLEWSKI ${ }^{a *}$, R. NARKoWICZ ${ }^{a, b}$, T. WojToWICZ ${ }^{a}$, G. KARCZEWSKI ${ }^{a}$, J. Kossut ${ }^{a}$, J.P. Bergman ${ }^{c}$ AND B. MonemaR ${ }^{c}$

${ }^{a}$ Institute of Physics, Polish Academy of Sciences

Al. Lotników 32/46, 02-668 Warsaw, Poland

${ }^{b}$ Semiconductor Physics Institute, A. Gostauto 11, 2600 Vilnius, Lithuania

${ }^{\circ}$ Dept. Phys. \& Meas. Technol., Linköping Univ., 58183 Linköping, Sweden

Strong localization effects present in quantum well structures of $\mathrm{CdTe} /$ $\mathrm{CdMnTe}$ noticeably affect exciton dynamics and strength and character of exciton-phonon interaction. We show that the temperature dependences of the PL linewidth, PL peak wavelength and PL decay time strongly deviate from those expected for Wannier-excitons in structures with atomically smooth interfaces.

PACS numbers: 71.35.-y, 71.55.Jv, 73.20.Mf

Free excitons (FEs) in bulk crystals and in quantum well (QW) structures of III-V and II-VI semiconductors are treated as Wannier-Mott excitons and potential fluctuations present in a $\mathrm{QW}$ plane are assumed to introduce only a weak perturbation to their properties. Such approach correctly describes exciton properties in high quality QW structures of GaAs/AlGaAs [1]. The applicability of this model to QW structures of II-VI semiconductors is less obvious. Recently, we have shown that density of interface defects in $\mathrm{QW}$ structures of $\mathrm{ZnCdSe} / \mathrm{ZnSe}$ is at least two orders in magnitude larger than in the case of GaAs/AlGaAs system [2]. Such increased density of interface defects (mostly due to composition and QW width fluctuations) must result in increased localization effects and deviation from the model of Wannier-Mott-like FEs. In this work we analyse such possibility for QW structures of CdTe/CdMnTe.

For Wannier-Mott excitons their emission energy should follow temperature $(T)$ changes of the band gap energy $\left(E_{\mathrm{G}}\right)$. This dependence for CdTe bulk sample is described by the following empirical law:

$$
E_{\mathrm{G}}=1.6145-\frac{A T^{2}}{(B+T)}[\mathrm{eV}]
$$

with $A=4.87 \times 10^{-4} \mathrm{eV} / \mathrm{K}$ and $B=139 \mathrm{~K}$ [3]. In this work we test validity of such approach for a series of $\mathrm{CdTe} / \mathrm{CdMnTe} \mathrm{QW}$ structures described in Table. The experiments discussed here were performed for excitons in $10 \mathrm{~nm}$ QW,

*fax: 48-22-8430926, e-mail: godlew@ifpan.edu.pl 
TABLE

Description of $\mathrm{CdTe} / \mathrm{CdMnTe} \mathrm{QW}$ structures.

\begin{tabular}{c|c|c|c|c}
\hline \hline Sample & Substrate & Growth direction & QWs [nm] & $\begin{array}{c}\text { Mn fraction in } \\
50 \text { nm wide } \\
\text { CdMnTe barriers }\end{array}$ \\
\hline Sample \#1 & GaAs & $(001)$ & $10,6,4$ and 2 & $30 \% \mathrm{Mn}$ \\
Sample \#2 & GaAs & $(001)$ & $10,6,4$ and 2 & $68 \% \mathrm{Mn}$ \\
Sample \#3 & CdTe & $(001)$ & $10,6,4$ and 2 & $65 \% \mathrm{Mn}$ \\
Sample \#4 & InSb & $(111)$ & $10,4,2.5,1.2$ & $30 \% \mathrm{Mn}$ \\
Sample \#5 & CdTe & $(111)$ & $10,6,4$ and 2 & $20 \% \mathrm{Mn}$
\end{tabular}

where potential fluctuations are smaller than in narrower QWs and the effects due to the magnetic polaron formation are rather minor. Temperature dependences of emission energy $E(T)$, photoluminescence (PL) linewidth and PL kinetics in picoseconds time range were studied. The observed temperature dependences of excitonic PL energy, shown in Fig. 1, deviate from that in bulk CdTe sample (dash line).

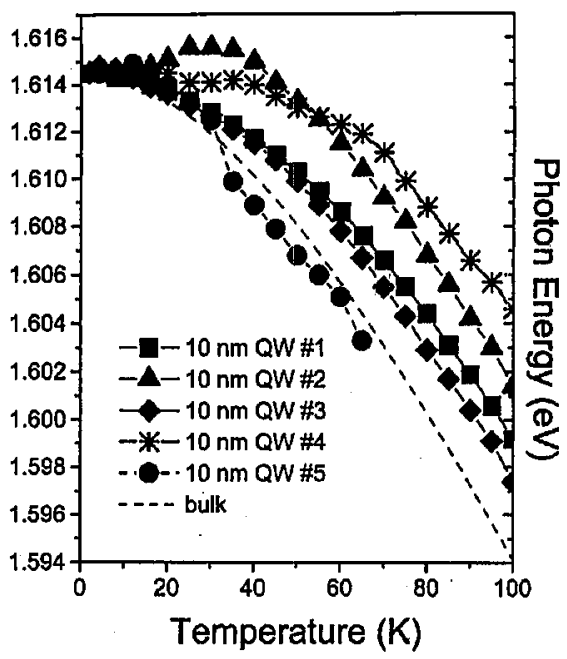

Fig. 1. Temperature dependence of PL emission energy of free excitonic emissions from $10 \mathrm{~nm}$ wide quantum wells in CdTe/CdMnTe structures described in Table.

Magnetic fluctuations were proposed to affect energy of magnetic CdMnTe system $[4,5]$. This contribution is negative and depends on temperature and magnetic susceptibility (Mn fraction). The effect should be small at low temperatures. A significant correction to the energy of the system is expected for relatively large Mn fractions in the CdMnTe barriers, for temperatures of $100 \mathrm{~K}$ and above and for relatively narrow $\mathrm{QWs}$, where the wave function of $\mathrm{FE}$ penetrates barrier regions. 
For all these reasons the magnetic fluctuations cannot account for the observed $E(T)$ dependences shown in Fig. 1. The present investigations indicate that strong localization effects (excitons properties deviate from those of Wannier-Mott free excitons) are important. FE energy follows the band gap changes of bulk CdTe only for the sample \#5, which is a homoepitaxial sample (CdTe substrate) with the lowest $\mathrm{Mn}$ fraction in the CdMnTe barriers. The largest deviation in exciton $E(T)$ dependence is observed for the sample \#4 grown on (111)InSb substrate. For this sample we observed the longest $2 \mathrm{~K} \mathrm{PL}$ decay time and the largest Stokes shifts, which indicates the very pronounced localization effects [6]. For the samples grown on either CdTe or GaAs substrate the deviation from $E(T)$ dependence of bulk CdTe increases with increasing $\mathrm{Mn}$ fraction in the CdMnTe barriers (structures \#2 and \#3). Time-resolved PL and PL excitation investigations indicate an increased roughness of $\mathrm{CdTe} / \mathrm{CdMnTe}$ interfaces in these samples. The strong localization effects are thus proposed to account for the observed $E(T)$ dependences of excitons in the samples \#2 and \#3.

Width and decay time of PL lines of Wannier-excitons should increase and PL coherence time should decrease [7-9]. This, at low temperatures, is due to scattering with acoustic phonons. Their occupation number can be approximated by a term linear with temperature. Thus the width of the PL lines and PL decay time should increase linearly with increasing temperature. Instead, we have observed temperature dependences, which strongly deviate from those expected for Wannier-excitons. This is observed even in the sample \#1, with relatively sharp PL lines and with the weak localization effects (Stokes shift of $0.5-1 \mathrm{meV}$ ). Width of the PL lines either weakly depends on temperature up to about $25-30 \mathrm{~K}$ or is practically temperature independent. In some cases (sample \#2) it slightly narrows before starting to increase. We explain the observed narrowing of the PL lines by a motional narrowing of the PL lines, which we expect for strongly localised excitons tunnelling between various localised sites by a phonon-assisted process. The process can be enhanced by interaction with hot carriers excited by microwave radiation. In the latter experiment carriers were heated at cyclotron resonance conditions, as proposed in Ref. [10].

To verify the pronounced role of localization effects, we performed PL kinetics measurements. In the case of large potential fluctuations in a QW plane, resulting in localization of excitons, the PL lines are inhomogeneously broadened and PL decay time is energy dependent. A drift of the exciton energy towards a lower energy is then observed during PL decay time [11]. For GaAs/AlGaAs QW structures such drift was observed only for the narrowest QWs and PL decay time was energy dependent only at the high-energy wing of the FE PL [11]. In contrast, for CdTe/CdMnTe structures with the largest Mn fractions in the CdMnTe barriers, exciton migration/tunnelling among localised states is observed even at the lowest temperatures and also for the widest QWs. In Fig. 2 we show a strong energy dependence of the PL kinetics observed for the $10 \mathrm{~nm}$ wide $\mathrm{QW}$ in the sample \#2. Due to the large density of localised states in this structure excitons do not thermalise within their decay time and energy dependent PL decay is observed. PL decay time is the shortest at the high-energy wing of the PL emission and varies within the whole PL line, i.e., the mobility edge is not observed. 


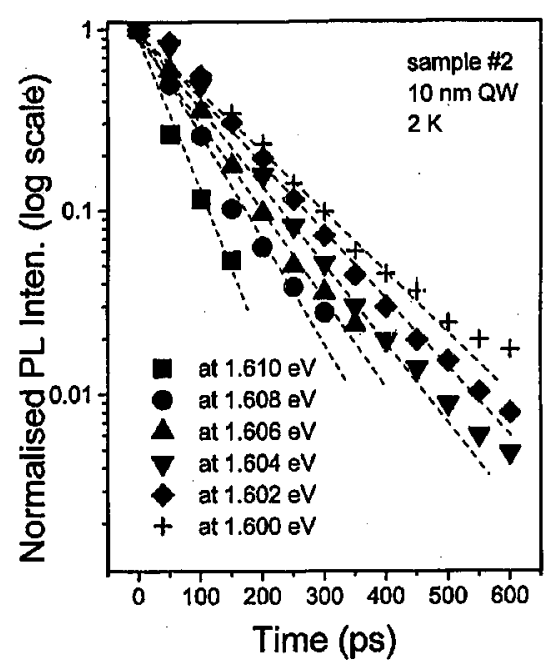

Fig. 2. Energy dependence of PL decay time within free excitonic emission from the $10 \mathrm{~nm}$ quantum well in CdTe/CdMnTe structure with $68 \% \mathrm{Mn}$ fraction in CdMnTe barriers. PL intensities at different energies within PL line were normalised to 1.

Concluding, in the CdTe/CdMnTe structures with large Mn fractions in the $\mathrm{CdMnTe}$ barriers, localization effects cannot be included as a weak perturbation of free exciton properties. Excitons are strongly localised and their properties differ from those of Wannier-Mott free excitons.

This work was partly supported by grants number 2P 03B 08709 and PBZ 28 11/P8 of the Committee for Scientific Research (Poland).

\section{References}

[1] M. Bugajski, M. Godlewski, J.P. Bergman, B. Monemar, K. Regiński, M. Kaniewska, Thin Solid Films 267, 84 (1995).

[2] M. Godlewski, J.P. Bergman, B. Monemar, E. Kurtz, D. Hommel, Appl. Phys. Lett. 69, 2843 (1996).

[3] B.S. Sundershesku, T. Kendelewicz, Phys. Status Solidi A 69, 467 (1982).

[4] J.A. Gaj, A. Golnik, Acta Phys. Pol. A 71, 197 (1987).

[5] R.B. Bylsma, W.M. Becker, J. Kossut; M. Dębska, Phys. Rev. B 33, 8207 (1986).

[6] M. Godlewski, J.P. Bergman, B. Monemar, Lithuanian Phys. J. 35, 563 (1995).

[7] T. Takagahara, J. Lumin. 44, 347 (1989).

[8] D.S. Citrin, Phys. Rev. B 47, 3832 (1993).

[9] M. Colocci, M. Gurioli, J. Martinez-Pastor, J. Phys. IV Collog. C 5, 3 (1993).

[10] M. Godlewski, W.M. Chen, B. Monemar, CRC Crit. Rev. Solid State Mater. Sci. 19, 241 (1994).

[11] M. Zachau, J.A. Kash, W.T. Masselink, Phys. Rev. B 44, 8403 (1991). 